\title{
Treatment of Adult Spondylolisthesis: PLIF versus PLF: Comparitive Study of Early Functional Outcome
}

\section{Sakthivel RN* and Balakrishnan V}

ESIC Medical College \& PGIMSR Chennai, Tamil Nadu, India

*Corresponding author: Rajarajan Nagu Sakthivel, ESIC Medical College \& PGIMSR Chennai, Tamil Nadu, India, Tel: 09841265183; E-mail: drnsrajarajan@yahoo.com

Received date: March 13, 2017; Accepted date: May 13, 2017; Published date: May 16, 2017

Citation: Sakthivel RN, Balakrishnan V (2017) Treatment of Adult Spondylolisthesis: PLIF versus PLF: Comparitive Study of Early Functional Outcome. J Clin Exp Orthop 3: 36. doi:10.21767/2471-8416.100036

Copyright: (c) 2017 Sakthivel RN, et al. This is an open-access article distributed under the terms of the Creative Commons Attribution License, which permits unrestricted use, distribution, and reproduction in any medium, provided the original author and source are credited.

\section{Abstract}

Objective: Aim of this study is to assess and compare the early functional outcomes and results of posterior lumbar interbody fusion (PLIF), and posterolateral fusion (PLF) in the surgical treatment of adult spondylolisthesis.

Background: Posterolateral fusion has been considered the best method for surgical treatment of adult spondylolisthesis. This technique had been widely used. Superior results have subsequently been reported with interbody fusion with cages and posterior instrumentation. In axial loading pull out strength and eccentric loading remains a disadvantage for PLF. The problems such as axial pain, loss of reduction, loosening or failure of fixation materials occurred relatively frequently after posterolateral fusion could be improved by an operation that fused vertebral bodies.

Materials and Methods: Fourty two patients with lumbar spondylolisthesis were operated with moderate to severe low back pain and/or sciatica. Patients with neurogenic claudication also were included. Randomisation technique was followed to allot patients into two different groups according to the mode of bony fusion into PLIF and PLF groups. Both groups were statistically identical.

Results: PLIF and PLF groups had no major differences in blood loss, short-term postoperative clinical result, or complications. Surgical time was slightly prolonged in PLIF when compared to PLF. Visual analog scale for back pain and leg pain, the Oswestry disability index, and fusion rates were significantly better in PLIF. The lumbar lordosis and the segmental angle revealed greater improvement in the PLIF group.

Conclusion: PLIF seems to be a better bone fusion technique than PLF in the management of lumbar spondylolisthesis. The problems encountered in PLF technique have been improved in PLIF.

Keywords: Spondylolisthesis; Spinal fusion; Posterior lumbar interbody fusion; posterolateral fusion

\section{Introduction}

The primary objective in spondylolisthesis is to achieve a stable situation in terms of construct either with or without instrumentation along with adequate decompression. This would help the patients feel symptomatic relief. The primary indication for surgery is unstable progressive listhesis with or without symptoms. Higher grades of listhesis have to be considered for surgery. Although there are many techniques ideal method still needs lot of research [1].

Hadra in 1889 was the first man to describe wiring for spondylolisthesis. On lay grafting was described by Albee [2] and Hibbs [3] using autologous cancellous grafts for tuberculosis of spine. Results were average with high incidence of pseudarthrosis. Later posterolateral fusion has shown good results and was widely used because of good results and less complications. Cloward [4] was the first to fuse two adjacent vertebral bodies using bone grafts. He also performed posterior laminectomy to decompress the cord. Cages were used to fuse two adjacent vertebras by Brantigan [5]. The material was with carbon.

To date, both PLF and PLIF are widely used fusion techniques, applied during spondylolisthesis surgery [6-8]. PLIF had been proved to be superior to PLF in certain studies $[6,7,9-16]$ whereas certain studies show that PLF is better $[8,10,11]$. There is a definitive indication to compare both the techniques which is the primary aim of our study.

\section{Materials and Methods}

42 cases with spondylolisthesis were taken and were randomly allotted into two groups. All cases were prospectively followed and the early functional outcome assessed. Randomization was achieved with software (random-allocationsoftware.software.informer.com/2.0) and computer assisted. Inclusion criteria were severe low back ache not relieved after suitable trial of conservative treatment, associated with or without sciatica. Patients with severe spondylosis and asymptomatic cases were excluded. The cases in either group shared similar demography. 
In instrumented postero-lateral fusion group of 23 cases adequate decompression is achieved by modified Gill technique along with bone graft from lamina along with pedicle screw fixation. Out of 23 cases, 10 were males and 13 females with a mean age of 50.4 ( $38 \mathrm{y}$ to $64 \mathrm{y}$ ). Meyerding grading was used and classified accordingly as grade I in 6 cases, grade II in 11 cases and grade III in 6 cases.

In the PLIF group of 19 cases, the titanium cages in the serial dilated disc space was filled with autologous graft ( $360^{\circ}$ fusion) after decompression discectomy was successfully performed. There were 7 males and 12 females and the mean age of $52.6 y$ ( 40 y to $70 \mathrm{y}$ ). Meyerding grading was grade I in 2 cases, grade II in 9 cases and grade III in 8 cases.

Average time taken for surgery in PLF group is 100minutes, and in PLIF group is 135 minutes. All cases had a minimum of 22 months follow up. All cases were followed up in 3 monthly intervals and evaluated using visual analog scale and the scale taken at 6 months considered as early outcome and the scale taken at 22 months as late outcome.

The Oswestry disability index (ODI) questionnaire is an ordinal scale and is also used for evaluation of functional results. The outcome is added and multiplied by 2 with worst results being score 100 and best being score 0 .

AP and Lateral views were used for objective assessment of fusion. Bony fusion is considered accomplished when bony trabeculae crosses along the lines of fusion. The intersomatic fusion (PLIF) was established according to the radiological criteria described by Brantigan et al., [5]. The degree of posterolateral fusion (PLF) was determined employing the method used by Lenke et al., [17].

\section{Statistical Analysis}

The statistical method used was chi squared test. Statistical significance was set at $95 \%$ and a $p$ value $0.05 \%$.

\section{Functional Results}

The study included 17 males and 25 female patients, with mean ages of 50.4 years in the PLF group and $52.6 \mathrm{y}$ in the PLIF group. Both groups were statistically identical.

All cases were followed up for a minimum of 22 months. The mean follow-up period was 28.3 months (range, 22-36 month) for the PLIF group and 27.42 months (range, 22-38 month) for the PLF group; the difference between the two groups was not significant.

On average at the end of six months VAS score was assessed and showed improved leg pain from $72 \mathrm{~mm}$ preoperatively to 38 $\mathrm{mm}$, whereas back pain improved from $64 \mathrm{~mm}$ preoperatively to $32 \mathrm{~mm}$ in the PLF group and the PLIF leg pain from $76 \mathrm{~mm}$ to 40 $\mathrm{mm}$ and back pain $68 \mathrm{~mm}$ to $28 \mathrm{~mm}$, but the difference between the two groups was not significant. At the end of final follow up VAS score in PLIF groups improved to $32 \mathrm{~mm}$ and slightly worsened to $44 \mathrm{~mm}$ in PLF group, whereas back pain improved in PLIF to $26 \mathrm{~mm}$ and increased to $40 \mathrm{~mm}$ in the PLF group; both the groups showed significant difference in terms of VAS for back pain at final follow up. In PLF group both radiating leg pain and back pain worsened in late follow up. In PLIF group both showed significant improvement.

The PLF group had a mean preoperative ODI of 40.2 (range, 24-76) that was reduced to a mean of 24.2 in early follow up and 26.1 (range, 21-60) at the final postoperative follow-up. However, in the PLIF group, it was reduced from 44.5 (range, 26-67) preoperatively to 23.0 in early follow up and 16.2 (range, 10-36) in the late follow up. The difference between the two groups was significant results varied according to the degree of slip. In grade 2 or 3, we observed good or very good results in $82 \%$ of cases with PLIF, versus $46 \%$ in cases with PLF. Resumption of original work in the PLIF group (60\% at 8 months) was better than in the PLF group ( $55 \%$ at 10 months).

\section{Complications}

Per op complications were almost nil in PLF group. One case from PLIF group had moderate to high bleeding from epidural venous plexus which was controlled intraoperatively. Narrow canal posed a difficulty in one case. During post op period transient radiculopathy was noticed probably because of manipulation (while attempting to reduce the listhesis, the nerve root could have stretched) but resolved on its own within 3 months in the PLF group. Few cases required revision procedure for impending pseudarthrosis. Out of revision cases $25 \%$ belonged to PLIF group and $75 \%$ to PLF group. In their second surgery, PLIF was performed. The difference between PLIF and PLF was significant. Two of the PLF cases had loss of reduction (Figure 1).

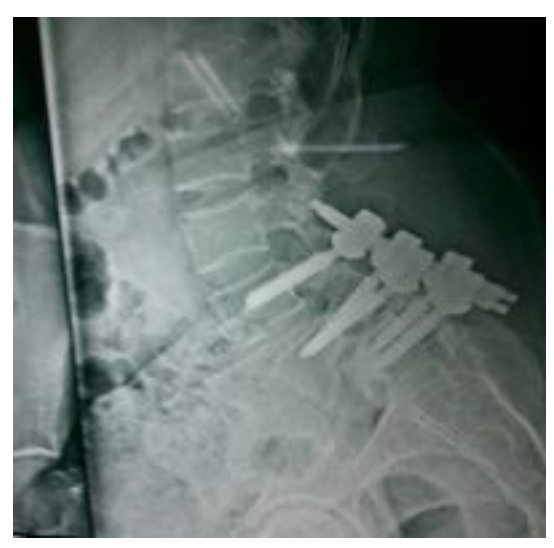

Figure1: Loss of reduction.

One patient had breakage of both S1 screws (Figure 2) under repeated stress because of failure of fusion which required implant removal. 

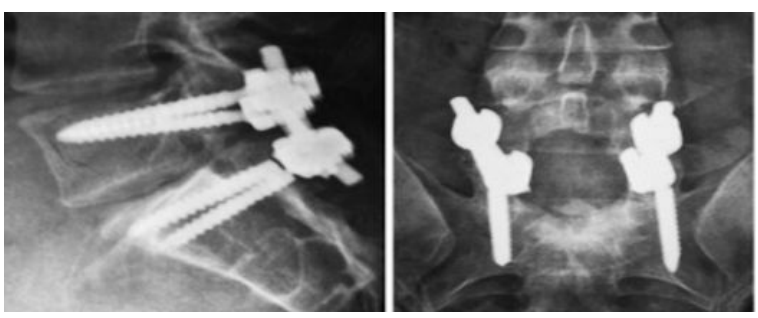

Figure 2: Screw breakage.

In the PLIF group 5 patients had mild radiculopathy which resolved on its own. Two patients from PLIF group and one patient in PLF group had acquired postoperative surgical infections which were treated with culture specific antibiotics.

\section{Radiological Results}

Radiological fusion was confirmed in 15 patients (79\%) of the PLIF group (Figure 3 ) and 12 patients (52\%) in the PLF group by the end of the first year. The difference in the prevalence of fusion between the two groups was significant (Figure 4).
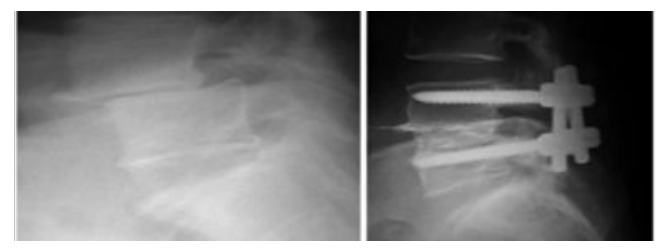

Figure 3: Radiological fusion.

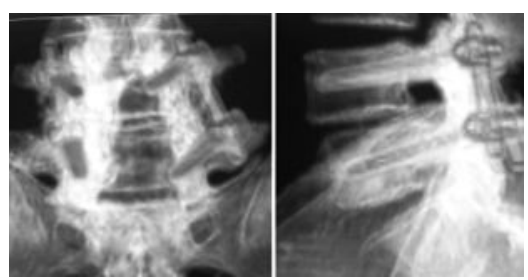

Figure 4: Difference in prevalence of fusion.

\section{Discussion}

Conservative treatment has shown good results in spondylolisthesis cases. But recently patient's life style and demands have shown more inclination towards surgery. The choice amongst surgical management is in debate now. PLF has shown good results in many studies but has more post-operative problems. PLIF is more advantageous in these situations. The quality of life of the patient is significantly improved after surgery. The progressive vertebral slipping is also avoided.

The mechanical instability in spondylolisthesis demands rigid fixation and solid fusion. Adequate decompression is the key factor in success. Rigid fusion between segments arrest further instability. As stated by Vibert [18], fusion is deferred only in cases with pre-existent spinal rigidity due to reactive sclerosis (Table 1).

Table 1: Measures of PLF and PLIF.

\begin{tabular}{|l|l|l|}
\hline Characteristics & PLF & PLIF \\
\hline Male/ Female & $10 / 13$ & $7 / 12$ \\
\hline Age & $38-64$ & $40-70$ \\
\hline Range & 50.4 & 52.6 \\
\hline Mean & 9 & \multicolumn{2}{|l|}{} \\
\hline Duration of symptoms & 14 & 13 \\
\hline$<1$ years & \multicolumn{2}{|l|}{} \\
\hline$>2$ years & 18 & 16 \\
\hline Surgery level & 5 & 3 \\
\hline One level & \multicolumn{2}{|l}{} \\
\hline Multi-level & $22-36$ & $22-38$ \\
\hline Follow up & \multicolumn{2}{|l|}{} \\
\hline Range(months) &
\end{tabular}

In the recent years surgical techniques and surgeon's skill in treating listhesis improved. This in turn has considerably increased the number of willing cases, while ensuring good clinical results. The best surgical technique still needs to have adequate research. While considering the Denis three columns concept the postero-lateral fusion aims to fuse only the posterior segment and thus less effective. Five of our cases from PLF group had implant failure and persistent symptoms. On retro analysis all the five cases had highly unstable segments with large disc space. The probable reason for this failure could be because PLF procedure controls only the posterior column while anterior biomechanics are not attended. But still some cases with grade I listhesis with stable looking segments and narrow space have shown excellent results in the PLF group (Table 2).

Table 2: Visual Analog Scale.

\begin{tabular}{|c|c|c|c|c|c|c|}
\hline & \multicolumn{2}{|c|}{ Pre OP } & \multicolumn{4}{c|}{ Post OP } \\
\hline & & & \multicolumn{2}{c|}{ Early FU } & \multicolumn{2}{c|}{ Late FU } \\
\hline & $\begin{array}{l}\text { Radiating } \\
\text { leg pain }\end{array}$ & $\begin{array}{l}\text { Back } \\
\text { pain }\end{array}$ & $\begin{array}{l}\text { Radiati } \\
\text { ng leg } \\
\text { pain }\end{array}$ & $\begin{array}{l}\text { Back } \\
\text { pain }\end{array}$ & $\begin{array}{l}\text { Radiati } \\
\text { ng leg } \\
\text { pain }\end{array}$ & $\begin{array}{l}\text { Back } \\
\text { pain }\end{array}$ \\
\hline $\begin{array}{l}\text { PL } \\
\text { F }\end{array}$ & 72 & 64 & 38 & 32 & 44 & 40 \\
\hline $\begin{array}{l}\text { PL } \\
\text { IF }\end{array}$ & 76 & 68 & 40 & 28 & 32 & 26 \\
\hline
\end{tabular}

The posterior lumbar intersomatic fusion (PLIF) allow us not only restoration of the disc height, but also improve the stability by decreasing the dead space and decompressing the dural sac and the roots. "Reduction in listhesis increases the disc space after PLF insertion of the cage improves the stability of the segment and also improves the lumbar lordosis and the segmental angle. 
This would demonstrate how the better results can be achieved with PLIF with respect to back pain. Restoration of native anatomy versus disc height, foraminal height, etc., are the prime success reasons behind PLIF.

Symptomatic improvement in the leg pain is also noticed. Majority of our cases had radiculopathy. In our series L5 and S1 nerve root involvement was seen in most of the cases.

Reactive fibro cartilaginous tissue mass formed because of instability at the pars inter-articularis compress the L 5/S 1 nerve root. Rattler laminar segment frequently irritates the dural sac and the nerve roots. Surgical decompression of the rattler lamina and the fibro cartilage mass at the defect in the pars interarticularis has given relief to both radicular pain and the low back pain. These compressing tissues of the neuroelements should be adequately decompressed for symptomatic relief. We have paid utmost attention for adequate surgical decompression (Table 3).

Table-3: Oswestry Disability Index.

\begin{tabular}{|l|l|l|l|}
\hline & Pre OP & Follow Up \\
\hline & & Early & Late \\
\hline PLF & 40.2 & 24.2 & 26.1 \\
\hline PLIF & 44.5 & 23.0 & 16.2 \\
\hline
\end{tabular}

In a study conducted by lee [19], both PLF and PLIF has shown good results with level 2 evidence. In another study by Ekman [20], after 2 year follow up both PLF and PLIF has shown only equal results. In long term cohort study by Cunningham [7], PLIF has shown superior results compared to PLF. In another study by Kee Yong [21] pre op instability is said to play a major role whether addition lumbar interbody fusion would be beneficial or not. Both PLF and PLIF are viable surgical options for patients with isthmic spondylolisthesis 60 years or younger.

In a study by Shalabi [22] adjacent segment degeneration was considered of prime importance and proved to be a factor in outcome. Compared to PLF only, PLF+ PLIF/TLIF were statistically significantly associated with a greater correction of spondylolisthesis. More number of patients in PLF group required revision surgeries than the PLIF group. Interbody fusion prevents progression of degenerative disease.

In another study by Pan Yong Fi [23] primarily PLIF is done when there is gross instability or severe canal stenosis requiring extensive laminectomy and decompression. Otherwise PLF has shown equal results. Another study by Babak [24] showed that TLIF is superior to PLF with respect to functional outcome and fusion rate. Low grade spondylolisthesis gives satisfactory results with respect to fusion in both PLF and PLIF techniques, but PLIF gives better results with respect to pain and stability.

\section{Conclusion}

PLIF procedure gives better clinical outcome and higher fusion rates and fewer implant failures than the PLF technique in the management of adult spondylolisthesis in this study.

\section{References}

1. Hadra BE (1975) The Classic. Wiring of the vertebrae as a means of immobilization in fracture and Pott's disease. Berthold E Hadra. Med Times and Register, Vol 22, May 23, 1891. Clin Orthop Relat Res 112: 4-8.

2. Albee FH (1911) Transplantation of a portion of the tibia into spine for Pott's disease. A Preliminary Report. JAMA 57: 885-887.

3. Hibbs RA (1988) A report of 59 cases of scoliosis treated by fusion operation. By Russell A Hibbs, 1924. Clin Orthop Relat Res 229: 4-19.

4. Cloward RB (1953) The treatment of ruptured lumbar intervertebral discs by vertebral body fusion. Indications, operative technique, after care. J Neurosurg 10: 154-168.

5. Brantingan JW, Steffe AD (1993) A carbon fiber implant to aid the interbody lumbar fusion: two year clinical results in the first 26 patients. Spine 18: 2106-2117.

6. Aoki Y, Yamagata M, Ikeda Y, Nakajima F, Ohtori S, et al. (2012) A prospective randomized controlled study comparing transforaminal lumbar interbody fusion techniques for degenerative spondylolisthesis: unilateral pedicle screw and 1 cage versus bilateral pedicle screws and 2 cages. J Neurosurg Spine 17: 153-159.

7. Cunningham JE, Elling EM, Milton AH, Robertson PA (2013) What is the optimum fusion technique for adult isthmic spondylolisthesis - PLIF or PLF? A long-term prospective cohort comparison study. J Spinal Disord Tech 26: 260-267.

8. Ekman P, Moller H, Tullberg T, Neumann P, Hedlund R (2007) Posterior lumbar interbody fusion versus posterolateral fusion in adult isthmic spondylolisthesis. Spine (Phila Pa 1976) 32: 2178-2183.

9. Hai Y, Kopacz KJ, Lee CK (1997) Posterior lumbar interbody fusion with and without pedicle screw fixation: comparison for clinical and radiological results. Presented at the North American Spine Society; New York, NY, October 22-25.

10. Han X, Zhu Y, Cui C, Wu Y (2009) A meta-analysis of circumferential fusion versus instrumented posterolateral fusion in the lumbar spine. Spine (Philadelphia) 34: E618-E625.

11. Kim KT, Lee SH, Lee YH, Bae SC, Suk KS (2006) Clinical outcomes of 3 fusion methods through the posterior approach in the lumbar spine. Spine (Phila Pa 1976) 31: 1351-1357.

12. Lee DY, Lee SH, Maeng PH (2010) Two-level anterior lumbar interbody fusion with percutaneous pedicle screw fixation: a minimum 3-year follow-up study. Neurol Med Chir (Tokyo) 50: 645-650.

13. Ray CD (1997) Threaded titanium cages for lumbar interbody fusions. Spine (Phila Pa 1976) 22: 667-680.

14. Steffee A, Sitkowski D (1988) Posterior lumbar interbody fusion and plates. Clin Orthop Relat Res 227: 99-102.

15. Fairbank JC, Pynsent PB (2000) The Oswestry disability index. Spine 25: 2940-2952.

16. Fairbank JC, Couper J, Davies JB, O' Brien JP (1980) The Oswestry Low Back Pain Disability Questionnaire. Physiotherapy 66: 271-273.

17. Lenke LG, Bridwell KH, Bullis D, Betz RR, Baldus C, et al. (1992) Results of In Situ Fusion for Isthmic Spondylolisthesis. J Spinal Disord 5: 433-442. 
18. Vibert BT, Sliva CD, Herkowitz HN (2006) Treatment of instability and spondylolisthesis. Surgical versus nonsurgical treatment. Clin Orthop Relat Res 443: 222-227.

19. Lee GW, Lee SM, Ahn MW, Kim HJ, Yeom JS (2014) Comparison of Posterolateral Lumbar Fusion and Posterior Lumbar Interbody Fusion for Patients Younger Than 60 Years With Isthmic Spondylolisthesis. Spine 15 39: E1475-E1480.

20. Ekman P Möller H, Tullberg T, Neumann P, Hedlund R (2007) Posterior Lumbar Interbody Fusion versus Posterolateral Fusion in Adult Isthmic Spondylolisthesis. Spine: 32: 2178-2183.

21. Ha KY, Na KH, Shin JH, Kim KW (2008) Comparison of Posterolateral Fusion With and Without Additional Posterior
Lumbar Interbody Fusion for Degenerative Lumbar Spondylolisthesis. J Spinal Disord Tech 21: 229-234.

22. Ekman P, Möller H, Shalabi A, Yu YX, Hedlund R (2009) A prospective randomised study on the long-term effect of lumbar fusion on adjacent disc degeneration. Eur Spine 18: 1175-1186.

23. Yongfei $\mathrm{P}$, Zubin $\mathrm{M}$, Shaodong $Z$, Xiaotao $\mathrm{W}$, Yonggang $L$, et al. (2011) United PLF and PLIF PLIF simple clinical treatment of lumbar degenerative disease Comparative study.

24. Alijani B, Emamhadi M, Behzadnia H, Azar M, Kazemnejad-Leili E, et al. (2015) Curb the pain of spondylolistehsis: comparing postero lateral fusdion versus posterior lumbar interbody fusion. IrJNS 1: 22-26. 